\title{
Studies of hadronic B decays to final states containing open charm mesons at LHCb
}

\section{Mark Whitehead ${ }^{* \dagger}$}

University of Warwick, $U K$

E-mail: mwhitehedcern.ch

\begin{abstract}
The latest results from the programme of studies of beauty hadron decays into final states with open charm at the LHCb experiment are presented. These decays provide several complementary measurements of $C P$ violation and parameters of the CKM matrix. The results in this report include first observations as well as some world leading measurements.
\end{abstract}

The European Physical Society Conference on High Energy Physics

18-24 July, 2013

Stockholm, Sweden

\footnotetext{
${ }^{*}$ Speaker.

On behalf of the LHCb Collaboration.
} 


\section{Introduction}

The LHCb experiment [1] is a single arm spectrometer, based at the Large Hadron Collider at CERN. It is optimised for the study of $b$ - and $c$-hadron decays, possessing excellent vertex resolution, particle identification from two RICH detectors and an efficient hadronic trigger. Decays of $b$-hadrons to open charm final states are an excellent place to study $C P$ violation, and in particular the angle $\gamma$ of the Unitarity Triangle.

This document details three independent measurements from $\mathrm{LHCb}$, each using a dataset corresponding to $1.0 \mathrm{fb}^{-1}$ of $p p$ collision data collected during 2011 . The following decays are reconstructed using the decay $D^{0} \rightarrow K^{-} \pi^{+}$only and charge conjugation is implied throughout.

\section{The first observation of $B_{s}^{0} \rightarrow \bar{D}^{0} \phi$ decays}

It has been shown in Refs. [2-5] that $B_{s}^{0} \rightarrow \bar{D}^{0} \phi$ decays can be used to measure the angle $\gamma$ using both time-integrated and time-dependent techniques. Additionally, the time-dependent approach provides sensitivity to $\beta_{s}$. The first step is to observe the decay mode for the first time. The decay proceeds via $b \rightarrow c$ and $b \rightarrow u$ tree-level amplitudes, resulting in a theoretically clean dependence on $\gamma$.

The analysis is based around a multi-variate selection, utilising the boosted decision tree method (BDT) [6]. The BDT is trained using simulation for the signal sample and events from the $D$ sidebands for the background sample. The decay $B_{s}^{0} \rightarrow \bar{D}^{0} \bar{K}^{* 0}$ is used as a normalisation channel to reduce systematic uncertainties. The LHCb measurement of $\mathscr{B}\left(B_{s}^{0} \rightarrow \bar{D}^{0} \bar{K}^{* 0}\right)$ [7] is also updated, using the decay $B^{0} \rightarrow \bar{D}^{0} K^{* 0}$ as a second control channel. Extended unbinned maximum likelihood fits are performed to extract the signal yields, as shown in Fig. 1. The number of signal events is $43 \pm 8$ for $B_{s}^{0} \rightarrow \bar{D}^{0} \phi, 535 \pm 30$ for $B_{s}^{0} \rightarrow \bar{D}^{0} \bar{K}^{* 0}$ and $260 \pm 24$ for $B^{0} \rightarrow \bar{D}^{0} K^{* 0}$. To check that the decay proceeds mainly via the $\mathrm{P}$-wave $B_{s}^{0} \rightarrow \bar{D}^{0} \phi$ channel and that the $\mathrm{S}$-wave
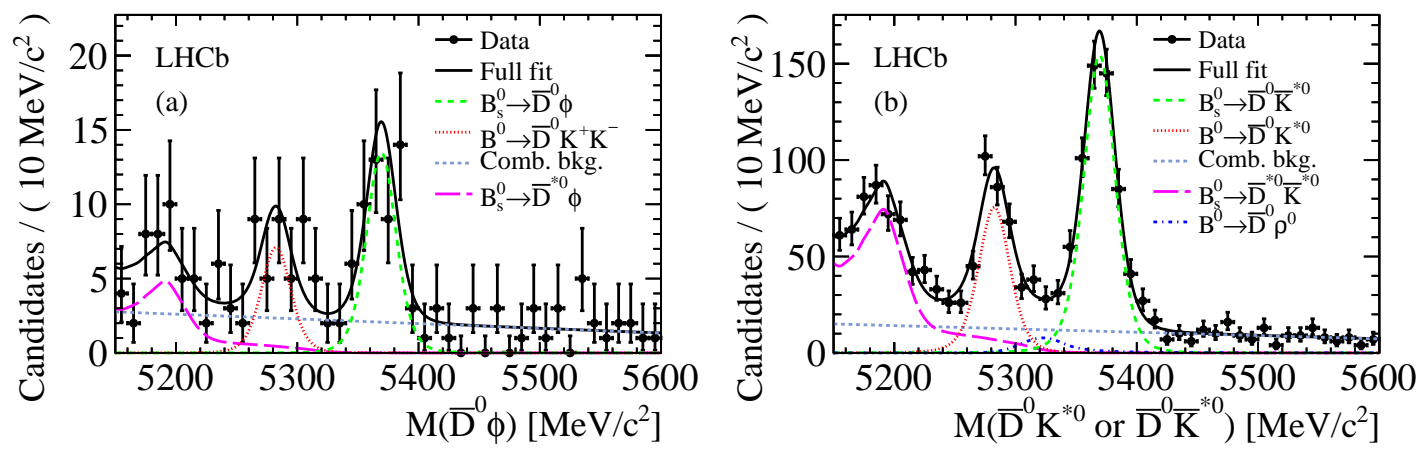

Figure 1: The $B$ candidate invariant mass distribution for (a) $B_{s}^{0} \rightarrow \bar{D}^{0} \phi$ and (b) $B_{(s)}^{0} \rightarrow \bar{D}^{0} \bar{K}^{* 0}$ candidates with the fit overlayed.

$B_{(s)}^{0} \rightarrow \bar{D}^{0} K^{+} K^{-}$contribution is small, the cosine of the helicity angle of the $\phi$ candidates, $\cos \theta_{\mathrm{h}}$, is inspected, as shown in Fig. 2. The simulated shape (histogram) shows the expected distribution of $\cos \theta_{\mathrm{h}}$ for events proceeding via the vector $\phi$ resonance. The $B_{s}^{0} \rightarrow \bar{D}^{0} \phi$ signal candidates (filled circles) clearly favour the simulated shape (black) compared to the S-wave distribution of the $B^{0} \rightarrow \bar{D}^{0} K^{+} K^{-}$candidates (triangles). 


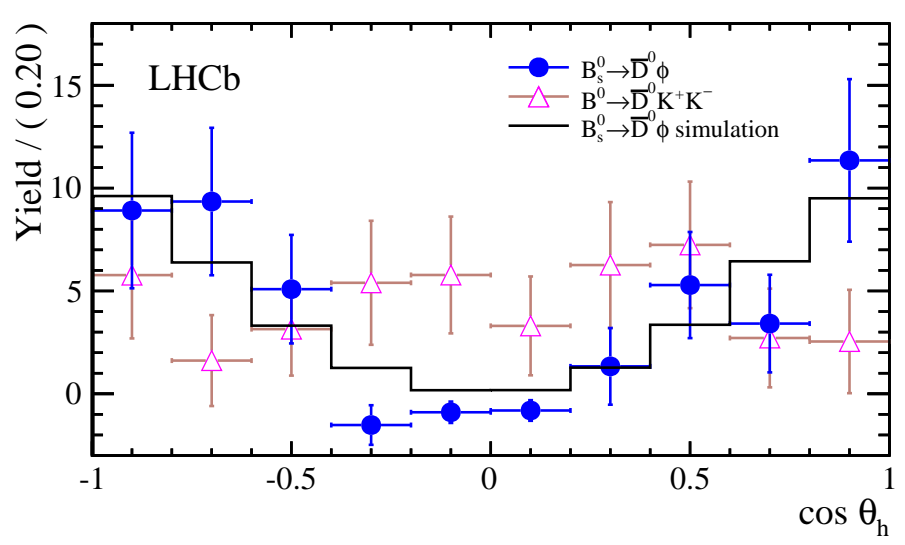

Figure 2: The cosine of the helicity angle of the $\phi$ candidates.

The branching fraction ratio is then determined to be

$$
\frac{\mathscr{B}\left(B_{s}^{0} \rightarrow \bar{D}^{0} \bar{K}^{* 0}\right)}{\mathscr{B}\left(B^{0} \rightarrow \bar{D}^{0} K^{* 0}\right)}=7.8 \pm 0.7(\text { stat }) \pm 0.3(\text { syst }) \pm 0.6\left(f_{s} / f_{d}\right),
$$

where $f_{s} / f_{d}$ is the ratio of fragmentation fractions with a value of $0.256 \pm 0.020$ [8]. Using the known value for $\mathscr{B}\left(B^{0} \rightarrow \bar{D}^{0} K^{* 0}\right)$ [9] gives

$\mathscr{B}\left(B_{s}^{0} \rightarrow \bar{D}^{0} \bar{K}^{* 0}\right)=\left[3.3 \pm 0.3(\right.$ stat $) \pm 0.1($ syst $\left.) \pm 0.3\left(f_{s} / f_{d}\right) \pm 0.5\left(\mathscr{B}\left(B^{0} \rightarrow \bar{D}^{0} K^{* 0}\right)\right)\right] \times 10^{-4}$.

This is the most accurate measurement of this branching fraction. The ratio of branching fractions for the signal decay is then determined to be

$$
\frac{\mathscr{B}\left(B_{s}^{0} \rightarrow \bar{D}^{0} \phi\right)}{\mathscr{B}\left(B_{s}^{0} \rightarrow \bar{D}^{0} \bar{K}^{* 0}\right)}=0.069 \pm 0.013(\text { stat }) \pm 0.007(\text { syst }) .
$$

Using the updated value for $\mathscr{B}\left(B_{s}^{0} \rightarrow \bar{D}^{0} \bar{K}^{* 0}\right)$ from above gives

$$
\mathscr{B}\left(B_{s}^{0} \rightarrow \bar{D}^{0} \phi\right)=\left[2.3 \pm 0.4(\text { stat }) \pm 0.2(\text { syst }) \pm 0.5\left(\mathscr{B}\left(B_{s}^{0} \rightarrow \bar{D}^{0} \bar{K}^{* 0}\right)\right)\right] \times 10^{-5} .
$$

This measurement represents an important first step in using this decay channel to measure the $C P$ violating phases $\gamma$ and $\beta_{s}$. The significance of this first observation is $6.2 \sigma$. For further details on this analysis please see Ref. [10].

\section{Measurement of the $B_{(s)}^{0} \rightarrow \bar{D}^{0} K^{-} \pi^{+}$branching fractions}

The decay $B^{0} \rightarrow \bar{D}^{0} K^{+} \pi^{-}$has been suggested $[11,12]$ as a channel that can play a significant role in the determination of the angle $\gamma$. To perform the necessary Dalitz plot analysis, the backgrounds to the decay mode must be well understood. Two potential backgrounds are $B_{s}^{0} \rightarrow \bar{D}^{0} K^{-} \pi^{+}$and, more seriously, $B_{s}^{0} \rightarrow \bar{D}^{* 0} K^{-} \pi^{+}$. Therefore, the first observation of $B_{s}^{0} \rightarrow \bar{D}^{0} K^{-} \pi^{+}$decays and knowledge of the Dalitz plot structure of the $B^{0} \rightarrow \bar{D}^{(*) 0} K^{+} \pi^{-}$mode are important. 
The decay $B^{0} \rightarrow \bar{D}^{0} \pi^{+} \pi^{-}$is chosen as the control sample to reduce systematic uncertainties. The control mode has very similar kinematics to the signal modes, allowing it to be used to train a neural network based on kinematic variables. NeuroBayes [13] was chosen as the neural network package for its ability to remain unbiased and not over-trained when training on data. The network is trained using $B^{0} \rightarrow \bar{D}^{0} \pi^{+} \pi^{-}$data and is optimised to observe the decay $B_{s}^{0} \rightarrow \bar{D}^{0} K^{-} \pi^{+}$. Extended unbinned maximum likelihood fits are performed to the $B$ candidate invariant mass distributions, as shown in Fig. 3. The signal yields are $8558 \pm 134$ for $B^{0} \rightarrow \bar{D}^{0} \pi^{+} \pi^{-}, 2391 \pm 81$ for $B_{s}^{0} \rightarrow \bar{D}^{0} K^{-} \pi^{+}$and $815 \pm 55$ for $B^{0} \rightarrow \bar{D}^{0} K^{+} \pi^{-}$decays. The ratio of branching fractions is
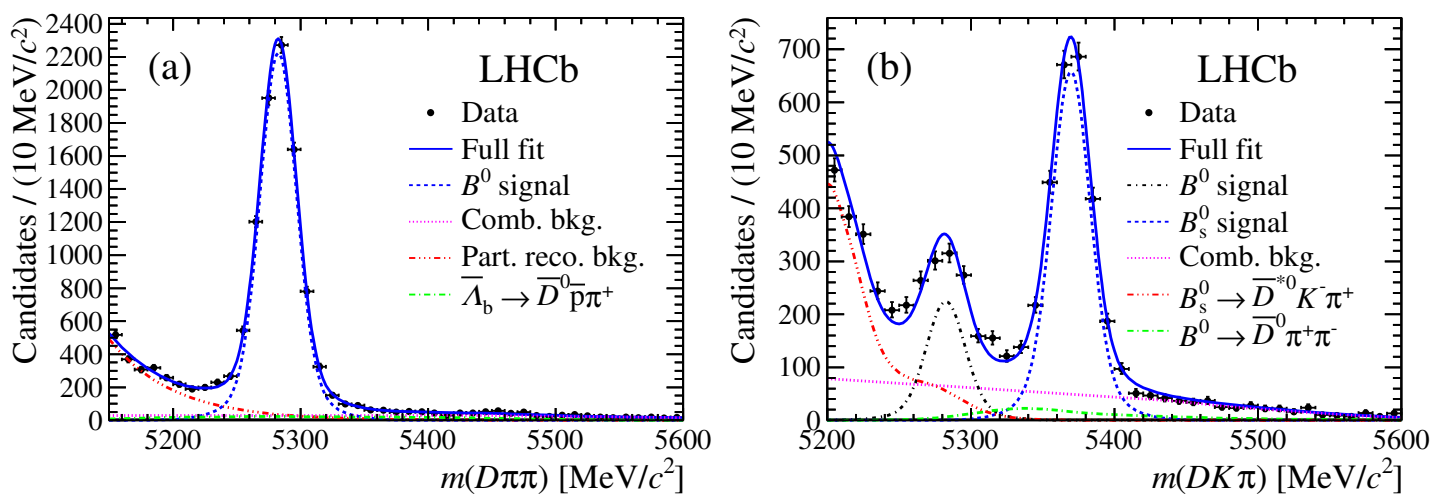

Figure 3: The $B$ candidate invariant mass distribution for (a) $B^{0} \rightarrow \bar{D}^{0} \pi^{+} \pi^{-}$and (b) $B_{(s)}^{0} \rightarrow \bar{D}^{0} K^{-} \pi^{+}$ candidates with the fit overlayed.

determined for the $B_{s}^{0} \rightarrow \bar{D}^{0} K^{-} \pi^{+}$decay to be

$$
\frac{\mathscr{B}\left(B_{s}^{0} \rightarrow \bar{D}^{0} K^{-} \pi^{+}\right)}{\mathscr{B}\left(B^{0} \rightarrow \bar{D}^{0} \pi^{+} \pi^{-}\right)}=1.18 \pm 0.05 \text { (stat) } \pm 0.12 \text { (syst), }
$$

where the systematic uncertainty includes the contribution from $f_{s} / f_{d}$. Using the measured value of $\mathscr{B}\left(B^{0} \rightarrow \bar{D}^{0} \pi^{+} \pi^{-}\right)[9]$ gives

$$
\mathscr{B}\left(B_{s}^{0} \rightarrow \bar{D}^{0} K^{-} \pi^{+}\right)=\left[1.00 \pm 0.04(\text { stat }) \pm 0.10(\text { syst }) \pm 0.10\left(\mathscr{B}\left(B^{0} \rightarrow \bar{D}^{0} \pi^{+} \pi^{-}\right)\right)\right] \times 10^{-3},
$$

which is the first observation of this inclusive decay with a significance in excess of $10 \sigma$. The ratio of branching fractions for the $B^{0} \rightarrow \bar{D}^{0} K^{+} \pi^{-}$decay mode has also been measured to be

$$
\frac{\mathscr{B}\left(B^{0} \rightarrow \bar{D}^{0} K^{+} \pi^{-}\right)}{\mathscr{B}\left(B^{0} \rightarrow \bar{D}^{0} \pi^{+} \pi^{-}\right)}=0.106 \pm 0.007 \text { (stat) } \pm 0.008 \text { (syst) }
$$

Using the known value for $\mathscr{B}\left(B^{0} \rightarrow \bar{D}^{0} \pi^{+} \pi^{-}\right)$this becomes

$$
\mathscr{B}\left(B^{0} \rightarrow \bar{D}^{0} K^{+} \pi^{-}\right)=\left[9.0 \pm 0.6(\text { stat }) \pm 0.7 \text { (syst) } \pm 0.9\left(\mathscr{B}\left(B^{0} \rightarrow \bar{D}^{0} \pi^{+} \pi^{-}\right)\right)\right] \times 10^{-3},
$$

which is the most precise measurement to date.

Additionally, the Dalitz plot distributions for signal events in the three decay modes are shown in Fig. 4. Please note that no amplitude analysis was undertaken, these plots were included for completeness and interest only. The visible resonances are (a) $\rho^{0}(770), f_{2}(1270)$ and $D_{2}^{*-}(2460)$; (b) $K^{* 0}(892)$ and $D_{2}^{*-}(2460)$, and (c) $K^{* 0}(892)$ and $D_{s 2}^{*-}(2573)$. Additional information on this analysis can be found in Ref. [14]. 

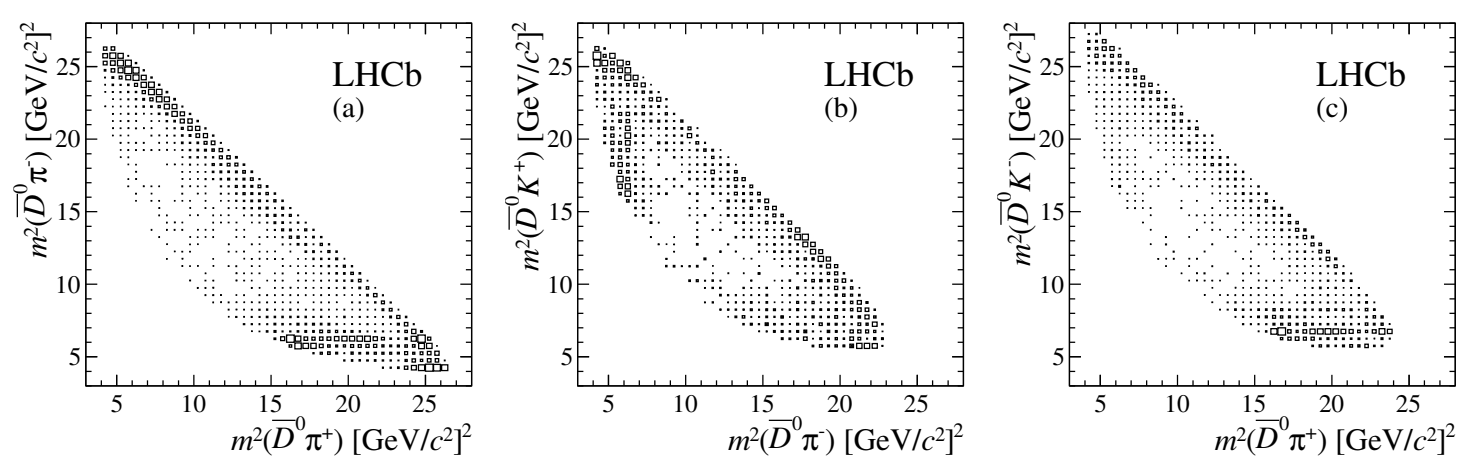

Figure 4: Signal weighted and efficiency corrected Dalitz plot distributions for (a) $B^{0} \rightarrow \bar{D}^{0} \pi^{+} \pi^{-}$, (b) $B^{0} \rightarrow \bar{D}^{0} K^{+} \pi^{-}$and (c) $B_{s}^{0} \rightarrow \bar{D}^{0} K^{-} \pi^{+}$candidates.

\section{Search for $B_{s}^{0} \rightarrow D^{*-} \pi^{+}$decays}

LHCb has recently measured decays such as $B_{s}^{0} \rightarrow D^{0} \bar{D}^{0}, D^{+} D^{-}$and $\pi^{+} \pi^{-}[15,16]$ for the first time. Such decays are mediated by weak-exchange and penguin annihilation processes, however the interplay of the two processes is not well understood. The scale of potential rescattering effects are also unknown. The decay $B_{s}^{0} \rightarrow D^{*-} \pi^{+}$is expected to be purely a weak-exchange process, so by measuring the branching fraction some theoretical models can be tested [17-19].

The $B^{0} \rightarrow D^{*-} \pi^{+}$decay mode is used as the control channel. It is also the main background, with the huge $B^{0}$ peak swamping the $B_{s}^{0} \rightarrow D^{*-} \pi^{+}$signal region. To control this effect, the data sample was binned in the angle $\theta_{\text {bach }}$, the angle between the $D^{*-}$ and the bachelor $\pi$ in the lab frame. This variable is strongly correlated to the width of the $B^{0}$ peak and provides a $20 \%$ gain in sensitivity. A simultaneous fit to the $B$ candidate invariant mass distribution is performed in five bins of $\theta_{\text {bach }}$, as shown in Fig. 5.
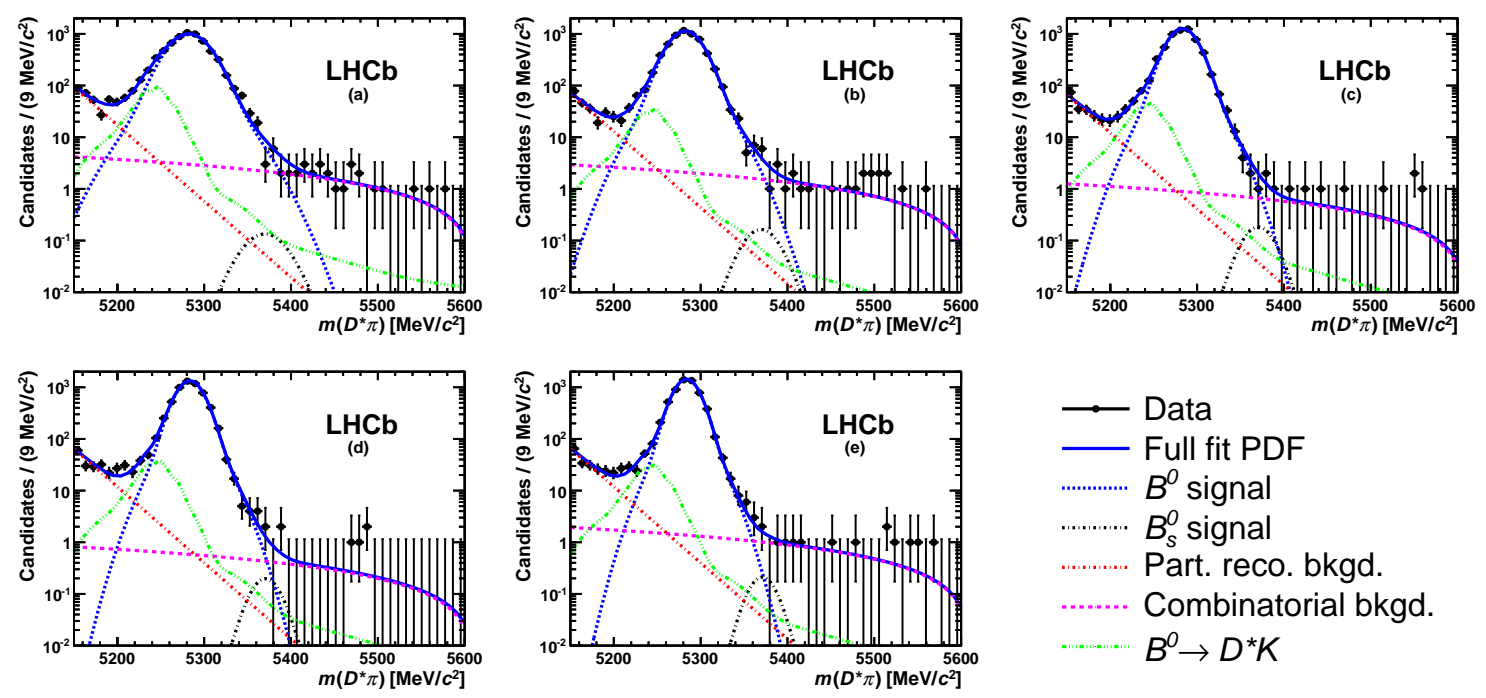

Figure 5: A simultaneous fit to the $B$ candidate invariant mass distribution in the following bins of $\theta_{\text {bach }}$; (a) 0-0.046, (b) 0.046-0.067, (c) 0.067-0.092, (d) 0.092-0.128 and (e) 0.128-0.4 rad. 
No significant signal was found, so an upper limit was set using a Bayesian method to be

$$
\mathscr{B}\left(B_{s}^{0} \rightarrow D^{*-} \pi^{+}\right)<6.1(7.8) \times 10^{-6} \text { at } 90 \%(95 \%) \mathrm{CL} .
$$

This represents the first limit on the branching fraction of this decay. The lack of a significant signal supports the prediction [19] that rescattering effects may make significant contributions to decays such as $B_{s}^{0} \rightarrow D^{0} \bar{D}^{0}, D^{+} D^{-}$and $\pi^{+} \pi^{-}$. Full details of the analysis are described in Ref. [20].

\section{Summary}

The first observation of the decay $B^{0} \rightarrow \bar{D}^{0} \phi$ has been presented with a significance of $6.2 \sigma$. The first observation of the inclusive channel $B_{s}^{0} \rightarrow \bar{D}^{0} K \pi$ had been made with a significance $>10 \sigma$ and the measurement of $\mathscr{B}\left(B^{0} \rightarrow \bar{D}^{0} K \pi\right)$ was updated. These results provide the building blocks for future analyses to study $C P$ violation and the angle $\gamma$. Finally, a search for $B_{s}^{0} \rightarrow D^{*-} \pi^{+}$decays was performed and an upper limit was set which provides an important input to understanding the dynamics of decays such as $B_{s}^{0} \rightarrow D^{0} \bar{D}^{0}$.

\section{Acknowledgements}

I would like to thank the European Research Council (ERC) for providing the funding that supports my position. I also wish to thank the members of the LHCb collaboration for their help in preparing this talk.

\section{References}

[1] LHCb Collaboration, J. Alves, A. Augusto et al. JINST 3 (2008) S08005.

[2] S. Nandi and D. London Phys.Rev. D85 (2012) 114015, [arXiv:1108.5769].

[3] M. Gronau and D. London Phys.Lett. B253 (1991) 483-488.

[4] M. Gronau, Y. Grossman, N. Shuhmaher, A. Soffer, and J. Zupan Phys.Rev. D69 (2004) 113003, [arXiv:hep-ph/0402055].

[5] M. Gronau, Y. Grossman, Z. Surujon, and J. Zupan Phys.Lett. B649 (2007) 61-66, [arXiv:hep-ph/0702011].

[6] L. Breiman, J. H. Friedman, R. A. Olshen, and C. J. Stone. Wadsworth international group, Belmont, California, USA, 1984.

[7] LHCb Collaboration, R. Aaij et al. Phys.Lett. B706 (2011) 32-39, [arXiv: 1110 . 3676 ].

[8] LHCb collaboration, R. Aaij et al. JHEP 1304 (2013) 001, [arXiv: 1301 . 5286].

[9] Particle Data Group, J. Beringer et al. Phys.Rev. D86 (2012) 010001.

[10] The LHCb collaboration, R. Aaij et al. arXiv: 1308.4583.

[11] T. Gershon Phys.Rev. D79 (2009) 051301, [arXiv:0810.2706].

[12] T. Gershon and M. Williams Phys.Rev. D80 (2009) 092002, [arXiv: 0909.1495 ].

[13] M. Feindt and U. Kerzel Nucl. Instrum. Meth. A 559 (2006), no. 1 190 - 194. 
[14] LHCb collaboration, R. Aaij et al. Phys. Rev. D 87, 112009 (2013), [arXiv:1304.6317].

[15] LHCb collaboration, R. Aaij et al. Phys.Rev. D87 (2013) 092007, [arXiv: 1302.5854 ].

[16] LHCb Collaboration, R. Aaij et al. JHEP 1210 (2012) 037, [arXiv: 1206.2794 ].

[17] M. Gronau, O. F. Hernandez, D. London, and J. Rosner Phys.Rev. D52 (1995) 6356-6373, [arXiv:hep-ph/9504326].

[18] R. Fleischer Nucl.Phys. B671 (2003) 459-482, [arXiv: hep-ph/0304027].

[19] M. Gronau, D. London, and J. L. Rosner Phys. Rev. D 87 (Feb, 2013) 036008.

[20] LHCb collaboration, R. Aaij et al. Phys.Rev. D87 (2013) 071101, [arXiv: 1302.6446 ]. 